\title{
Strategies for structure solution of small-molecule organics by 3D ED using a small beam
}

\author{
E. Mugnaioli ${ }^{1}$, I. Andrusenko', M. Gemmi ${ }^{1}$, P. P. Das ${ }^{2}$, A. S. Galanis², S. Nicolopoulos² \\ ${ }^{1}$ Center for Nanotechnology Innovation@NEST, Istituto Italiano di Tecnologia, Piazza San Silvestro 12, Pisa 56127, Italy, \\ ${ }^{2}$ NanoMEGAS SPRL, Rue Émile Claus 49 bte 9, Brussels1050, Belgium
}

enrico.mugnaioli@iit.it

Three-dimensional electron diffraction (3D ED) has matured into a method routinely employed by several worldwide-located laboratories for addressing crystallographic problems, which were considered intractable by X-ray diffraction [1]. The main advantage of ED is the ability to get diffraction data from volumes of few hundreds or even few tens of nanometers. This allows acquiring comprehensive 3D structural information from crystals too small for X-ray single-crystal methods, from coherent domains in pervasively twinned or disordered materials, from isolated domains embedded in inorganic or biological matrices and from minor constituents of powdered polyphasic mixtures.

From the beginning, the main shortcomings of 3D ED appeared connected with the deterioration of the sample induced by TEM vacuum or by beam damage. Moreover, organic crystals are typically affected by mosaicity and bending. In this contribution we will show different experimental protocols for data collection and analysis that can be employed in any experimental set-up, even for lowvoltage TEMs. Beam damage is minimised by coupling STEM imaging for sample search and tracking, and a small-size low-intensity parallel electron beam for diffraction data acquisition [2]. The size of the beam is crucial for picking narrow areas of the sample, either when coherent crystal domains are very small or when there is a need for moving to fresh parts of the crystal in order to lessen beam damage effects.

According with the specific sample characteristics, 3D ED data acquisition can be performed in stepwise mode, coupled with beam precession [3], or by continuous rotation [4]. Both approaches strongly benefit by the disposal of a new-generation ultra-fast singleelectron detector [5]. Moreover, background noise can be almost completely suppressed with an energy filter that cuts out the inelastic scattering. Structure solution is normally obtained ab-initio by direct methods. Still, global optimisation approaches, like simulated annealing [6], are valuable alternatives when data are affected by low resolution, preferential orientation or experimental errors that compromise the overall intensity reliability (e.g. beam damage, merohedric twinning, diffuse scattering).

We will thus discuss examples of recently published $[7,8]$ and forthcoming structure characterizations of pharmaceutical compounds, organic charge-transfer co-crystals and polycyclic aromatic hydrocarbons. For each case, specific problematics of the sample will be discussed, together with experimental solutions adopted for achieving structural solution and refinement.

[1] Gemmi, M., Mugnaioli, E., Gorelik, T. E., Kolb, U., Palatinus, L., Boullay, P., Hovmöller, S. \& Abrahams, J. P. (2019). ACS Cent. Sci. 5, 1315.

[2] Kolb, U., Gorelik, T., Kübel, C., Otten, M. T. \& Hubert, D. (2007). Ultramicroscopy 107, 507.

[3] Lanza, A., Margheritis, E., Mugnaioli, E., Cappello, V., Garau, G. \& Gemmi, M. (2019). IUCrJ 6, 178.

[4] Gemmi, M. \& Lanza, A. E. (2019). Acta Cryst. B75, 495.

[5] van Genderen, E., Clabbers, M. T. B., Das, P. P., Stewart, A., Nederlof, I., Barentsen, K. C., Portillo, Q., Pannu, N. S., Nicolopoulos, S., Gruene, T. \& Abrahams, J. P. (2016). Acta Cryst. A72, 236.

[6] Burla, M. C., Caliandro, R., Carrozzini, B., Cascarano, G. L., Cuocci, C., Giacovazzo, C., Mallamo, M., Mazzone, A. \& Polidori, G. (2015). J. Appl. Cryst. 48, 306.

[7] Andrusenko, I., Hamilton, V., Mugnaioli, E., Lanza, A., Hall, C., Potticary, J., Hall, S. R. \& Gemmi, M. (2019). Angew. Chem. Int. Ed. 58, 10919.

[8] Das, P. P., Andrusenko, I., Mugnaioli, E., Kaduk, J. A., Nicolopoulos, S., Gemmi, M., Boaz, N. C., Gindhart, A M. \& Blaton, T., (2021). Cryst. Growth Des. 21, 2019.

Keywords: electron diffraction; small-molecule organics; pharmaceutical compounds; beam damage; nanomaterials 